\title{
Behavioral representation in modeling and simulation introduction to CMOT special issue-BRiMS 2010
}

\author{
William G. Kennedy • Frank E. Ritter • \\ Bradley J. Best
}

Published online: 5 November 2011

(C) Springer Science+Business Media, LLC 2011

Keywords Cognitive modeling · Agent-based modeling $\cdot$ Social theory

The BRiMS Society and Conference (Behavioral Representation in Modeling and Simulation (BRiMS, brimsconference.org) promote cross-disciplinary communication for basic and applied scientific research in the realm of modeling and simulation of human behavior, with a particular emphasis on defense government-related tasks and behavior. Thus, the BRiMS conference brings together scientists, engineers, practitioners, and application users to discuss modeling behavior ranging from that of individuals to the behavior of whole societies, their interactions, and their implications. For a few days each year, we get to meet to share ideas and experiences, identify gaps in current capabilities, discuss new directions, highlight promising technologies, and showcase applications.

This special issue is similar to our previous special issue (Kennedy et al. 2010) in that it includes four papers based on the award winning conference papers of the 2010 annual conference, reviewed and extended to journal article length. The papers include a new model integrating top-down and bottom-up factors affecting visual target acquisition (Jungkunz and Darken 2011), the application of a statistical methodology

\footnotetext{
W.G. Kennedy $(\bowtie)$

George Mason University, 4400 University Drive, Fairfax, VA 22030-4400, USA

e-mail: wkennedy@gmu.edu
}

F.E. Ritter

Pennsylvania State University, 316G IST Building, University Park, PA 16802, USA

e-mail: frank.ritter@psu.edu

B.J. Best

Adaptive Cognitive Systems LLC, 1942 Broadway St., Suite 314, Bolder, CO 80302, USA

e-mail: bjbest@adcogsys.com 
to modeling psychological and cognitive impacts of protective clothing (Mueller et al. 2011), the use of fuzzy cognitive mapping techniques to model situation awareness (Jones et al. 2011), and the challenge of exploration and optimization of cognitive models (Moore 2011). Overall, they represent how the 2010 conference addressed modeling from small-scale models, for example, predicting eye movements, to largescale parameter exploration using high-performance computing facilities.

At the small-scale end of the range of these papers, Patrick Jungkunz and Christian Darken present models of eye movements during target acquisition in military simulations. They found that a relevance map performed better than a salience map and that scene locations that are semantically relevant predict human eye fixations better than just visual salience. However, the combined approach was not statistically better than the relevance map alone. Their work identified semantically relevant scene locations as the most significant factor in predicting eye fixations and they developed a novel method that supports direct extraction of that information directly from the simulation environment. This work is important for simulations because they too often assume that models can see everything (Ritter et al. 2000). Not seeing everything or seeing things that are trying not to be seen is important in adversarial simulations (Best and Gerhart 2011).

The second paper concerns the predicting the impact of the psychological and cognitive stressors on performance. Shane Mueller, Benjamin Simpkins, George Anno, Corey Fallon, Gene McCellan, and Owen Price of Applied Research Associates, report on the modification of an analysis technique to address the impact of a specific type of stressors on human performance. The stressors they studied were the restrictions of chemical protective clothing and gear used by military personnel. Adapting the task-taxon-task methodology allowed them to predict the performance degradation for a variety of tasks. This extends the IMPRINT approach that has been very successful in large system design, helping the Army save hundreds of millions of dollars (Booher and Minninger 2003).

Situation awareness is a critical component of military decision making at many levels of command. Our third paper describes the research behind a cognitive model that supports part of the decision-making process for infantry platoon leaders. Rashaad Jones, Erik Connors, Mary Mossey, John Hyatt, Neil Hansen, and Mica Endsley of SA Technologies, Inc., use a fuzzy cognitive mapping technique to develop a cognitive model that computationally represents infantry platoon leader situation awareness. In their paper, they describe the methodology to design and develop the model, and the validation process to assess the accuracy of the model's decisions. This is a nicely validated model, and helps explain situation awareness.

Finally, our last example from the 2010 BRiMS conference is a report by Richard Moore of Lockheed Martin Systems Management about his work at the Air Force Research Laboratory. He reports on his development of two algorithms to improve the computational challenges of exploring the enormous parameter space found in modeling and simulation efforts. Searching the parameter space of a cognitive model is not simply a task for large-scale computing environments. It requires an intelligent approach to be effective. Richard Moore has implemented two intelligent parallel search and exploration algorithms that reduce the computational costs of these efforts. Efforts like this will help us understand our models and architectures. 
These papers represent the range of work associated with the BRiMS 2010 conference. The BRiMS community, now a formalized society, has further raised the bar with the 2011 conference. Submissions scored better on average with reviewers, there were increased submissions and attendance, and there was increased international participation. Plenary speakers talked about applications, large-scale simulations (millions of un-encapsulated agents), social network theory, and more rational agents. These were indicative of what we, as a community, are increasingly able to develop: behavioral models and simulations employing more rational agents, agents able to do more complex tasks, and larger networks of agents. We have started the process of bringing the best of the BRIMS 2011 conference to a future issue of this journal.

\section{References}

Best BJ, Gerhart N (2011) An instance of what? Determining cues, attributes, and goals in a virtual task environment. In: Proceedings of the 20th conference on behavior representation in modeling and simulation (BRIMS). BRIMS Society, Sundance, pp 58-65

Booher HR, Minninger J (2003) Human systems integration in Army systems acquisition. In: Booher HR (ed) Handbook of human systems integration. Wiley, Hoboken, pp 663-698

Jones R, Connors ES, Mossey ME, Hyatt JR, Hansen NJ, Endsley MR (2011) Using fuzzy cognitive mapping techniques to model situation awareness for army infantry platoon leaders. Comput Math Org Theory (this special issue)

Jungkunz P, Darken CJ (2011) A computational model for human eye-movements in military simulations. Comput Math Org Theory (this special issue)

Kennedy WG, Ritter FE, Best BJ (2010) The best papers from BRIMS2009: cultural and group models. Comput Math Organ Theory 16(3):217-219

Moore LR (2011) Cognitive model exploration and optimization: a new challenge for computer science. Comput Math Org Theory (this special issue)

Mueller SG, Simpkins B, Anno G, Fallon CK, Price O, McClellan GE (2011) Adapting the task-taxon-task methodology to model the impact of chemical protective gear. Comput Math Org Theory (this special issue)

Ritter FE, Baxter GD, Jones G, Young RM (2000) Supporting cognitive models as users. ACM Trans Comput-Hum Interact 7(2):141-173

William G. Kennedy is a Research Assistant Professor with the Center for Social Complexity and the Department of Computational Social Science within the Krasnow Institute for Advanced Study at George Mason University. The majority of his research effort is on agent-based modeling (ABM) of conflict with agents representing the household level of societal aggregation. His research interests include: integrating computational cognitive modeling and computational social science; cognitive modeling of Theory of Mind phenomena; cognitive plausibility; and cognitive robotics. He is also a retired Navy Captain (submarine service) and a retired civil servant (Nuclear Regulatory Commission and Department of Energy). He was a member of the program committee for the 2011 Annual Meeting of the Cognitive Science Society and was a technical program co-chair for the BRiMS 2009, 2010, and 2011 conferences.

Frank E. Ritter is one of the founding faculty of the College of IST, an interdisciplinary academic unit at Penn State to study how people process information using technology. Frank Ritter's current research is in the development, application, and methodology of cognitive models, particularly as applied to interface design, predicting the effect of behavioral moderators, and understanding learning. He edits the Oxford Series on Cognitive Models and Architectures, is an editorial board member of Human Factors, Cognitive Systems Research, and the Journal of Educational Psychology, and recently was a technical program cochair for the BRiMS 2009, 2010, and 2011 conferences.

Bradley J. Best is a Principal Scientist at Adaptive Cognitive Systems, LLC, in Boulder, CO., where he focuses on modeling cognition and adaptive behavior in complex environments, especially those that 
have significant spatial and temporal aspects. His current research interests include integrating perception with decision making in robotic and virtual agents, with a focus on adversarial domains, and the development of methods for analyzing, understanding and visualizing model behavior in these environments. He was a member of the program committee for the 2010 and 2011 Annual Meetings of the Cognitive Science Society and was a technical program co-chair for the BRiMS 2009, 2010, and 2011 conferences. 